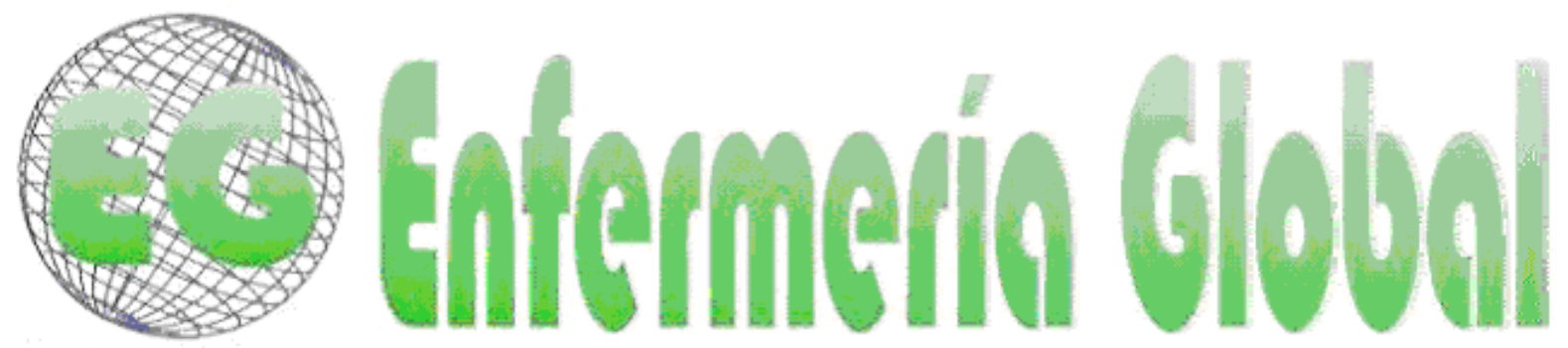

\title{
Estrategias para la reducción de la transmisión vertical del virus de inmunodeficiencia humana (VIH) y su relación con Enfermería
}

Estratégias para redução da transmissão vertical do vírus da imunodeficiência humana (HIV) e sua relação com a Enfermagem

\begin{abstract}
"Bernardes, MJC., ** Sousa Vilela, M., "**De Azevedo Filho, FM.
*Enfermero, Especialista en Terapia Intensiva. Profesor del Curso Técnico de Enfermería del Colégio Sena Aires, 2012. E-mail: milton.bernardes@hotamil.com ** Enfermera, Especialista en Saloud del Trabajador y profesora de práctica clínica del Colégio Sena Aires y Coordinadora del Programa de Planificaciónm Familiar de la Prefeitura de Aparecida de Goiânia, 2012.*** Enfermero, Especialista en Terapia Intensiva, Profesor adjunto de la Universidad Estadual de Goiás y Facultad Estácio de Sá, 2012. Brasil.
\end{abstract}

Palabras clave: Enfermería; VIH; Mujeres.

Palavras chave: Enfermagem; HIV; Mulheres.

Keywords: Nursing; HIV; Women.

\section{RESUMEN}

Introducción: Cerca del $40 \%$ de las personas infectadas por el vírus VIH son mujeres, feminización de la epidemia, ocurriendo de esta forma un aumento de casos de niños infectados a través de la TV.

Objetivos: Identificar las estrategias utilizadas en el proceso de investigación diagnóstica de la gestante y la importancia del enfermero en este proceso.

Material y Métodos: Se trata de una revisión bibliográfica en bases de datos virtuales.

Resultados y Discusión: Se encontraron 56 publicaciones, de las cuales tras realizar una lectura exploratoria de sus resúmenes, fueron seleccionados 17 artículos que contienen el tema propuesto. Se establecieron las siguientes estrategias: evaluación y educación sexual del $\mathrm{VIH}$, que consiste en la realización de exámenes gratuitos y consejos a las gestantes antes y después del test. Yen la adhesión a la terapia anti-retroviral, indicada para las gestantes seropositivas, percibimos que hay una gran dificultad en la continuidad del tratamiento, sin embargo los factores deben ser investigados individualmente. En estas estrategias el enfermero tiene un papel significativo en su ejecución..

Consideraciones finales: Son varios los desafios encontrados, siendo necesaria la constante actualización para la asistencia de enfermería, constituyendo la aplicación de acciones y de informaciones científicas un objetivo de la prevención y tratamiento para la disminución de la TV. 


\section{RESUMO}

Introdução: Cerca de $40 \%$ das pessoas infectadas pelo vírus HIV são mulheres, denominando a feminização da epidemia, ocorrendo desta forma um aumento de casos de crianças infectadas através da TV.

Objetivos: Identificar as estratégias utilizadas no processo de investigação diagnóstica da gestante e a importância do enfermeiro nesse processo.

Material e Métodos: Trata-se de um levantamento bibliográfico em base de dados virtuais.

Resultados e Discussão: Foram encontradas 56 publicações, dos quais após realizada uma leitura exploratória de seus resumos, foram selecionados 17 artigos que abrangem o tema proposto. Foram levantadas as seguintes estratégias: testagem e aconselhamento do HIV, que consiste na realização de exames gratuitos e aconselhamento das gestantes antes e após o teste. $E$ a adesão à terapia antiretroviral, indicada para as gestantes soropositivas, percebemos que há uma grande dificuldade na continuidade do tratamento, no entanto os fatores devem ser investigados individualmente. Sendo que em ambas as estratégias o enfermeiro encontra-se em um papel significativo para execução das mesmas.

Considerações finais: São vários os desafios encontrados, sendo necessária a constante atualização para a assistência de enfermagem, constituindo a aplicação de ações e de informações científicas com objetivo da prevenção e tratamento visando a diminuição da TV.

\section{ABSTRACT}

Introduction: About $40 \%$ of people infected with HIV are women, called the feminization of the epidemic, in this way an increase occurs in cases among children infected through TV. Objectives: To identify the strategies used in the diagnostic investigation of pregnancy and the importance of nurses in the process.

Methods: This is a bibliographic survey on the database software.

Results and Discussion: There were 56 publications, of which after reading the exploratory summaries, 17 articles were selected covering the proposed topic. The following strategies were raised: HIV testing and counseling, which consists of free testing and counseling and examinations of pregnant women before and after the test. Joining the antiretroviral therapy, given to the women who are pregnant and HIV positive, we realize that continuation of the treatment is very difficult, however the factors must be investigated individually. In both the strategies the nurse has in a significant role to play in the implementation of treatment.

Final considerations: There are several challenges encountered, there is a constant need to update the assistance of nursing and the implementation of actions and scientific information with the objective of prevention and treatment is aimed at the reduction of TV.

\section{INTRODUCCIÓN}

El Síndrome de Inmunodeficiencia Adquirida (AIDS) considerada como la más reciente pandemia de la humanidad, es actualmente uno de los más serios problemas de salud pública. Es una enfermedad crónica causada por el Virus de Inmunodeficiencia Humana (VIH), ocasionando pérdida progresiva de la inmunidad celular y, consecuentemente, la aparición de infecciones oportunistas ${ }^{1}$. 
Actualmente existen en el mundo cerca de 23 millones de personas infectadas por $\mathrm{VIH}$, de las que aproximadamente $40 \%$ son mujeres, caracterizando lo que se llamó feminización de la epidemia. Datos presentados por el Ministerio de Salud muestran que la epidemia de AIDS en Brasil continúa en niveles elevados, habiendo alcanzado en 2003 la incidencia de 18,4 casos por 100.000 habitantes. En los hombres hay una tendencia a la estabilización, registrándose una tasa menor que en 1998. Sin embargo, el crecimiento continúa entre las mujeres, siendo que en 2003 ocurrió la mayor tasa de incidencia en este grupo poblacional: 14,1 casos por 100.000 mujeres $^{1,2}$.

Consecuencia de esa feminización de la epidemia, ocurrió un aumento de casos de AIDS en niños, debido especialmente a la transmisión vertical (TV), o sea, la transmisión del virus al bebé durante la gestación, el parto o el amamantamiento materno. En Brasil, los datos epidemiológicos muestran que hasta junio de 2005, cerca de $83,7 \%$ de seropositividad del Virus de Inmunodeficiencia Humana $(\mathrm{VIH})$, en niños menores de 13 años, ocurrieron por la transmisión vertical. En el País, entre los 11.901 casos notificados de síndrome de imunodeficiencia adquirida (AIDS) en niños menores de trece años de edad, 9.965 ocurrieron por transmisión vertical ${ }^{1,3}$.

Estudios demuestran que varias mujeres toman conocimiento de la propia seropositividad cuando descubren que su hijo está infectado, o al realizar el pre-natal, o incluso durante el parto y en el postparto. El aumento de la transmisión vertical del VIH se presenta como un gran desafío para la salud pública, pues esta modalidad de transmisión es la principal vía de infección del VIH en la población infantil ${ }^{1,3,4}$.

Para reducir las tasas de transmisión vertical del VIH, Brasil adoptó como política pública el ofrecimiento del test anti-VIH a todas las gestantes durante los cuidados en el prenatal. Además de ofrecer el tratamiento a la gestante con la quimioprofilaxia, caso sea constatada su seropositividad, reduciendo de esta forma drásticamente el riesgo de transmisión del VIH para el bebé ${ }^{3}$.

Aunque haya en Brasil disponibilidad de tests y medicación que posibilitan tasas reducidas de transmisión vertical, según los datos epidemiológicos los niños continúan infectándose. Muchas mujeres llegan a las maternidades sin haber frecuentado el prenatal. De este modo, para la mayoría de las mujeres con $\mathrm{VIH}$, la única oportunidad de tener acceso al cuidado, al test para la investigación del $\mathrm{VIH}$ y al tratamiento quimioprofiláctico de la transmisión vertical es en el momento del parto y puerpério $^{56}$.

\section{OBJETIVO}

Ante lo expuesto, se objetivó a través de la literatura actual, identificar las estrategias utilizadas en el proceso de investigación diagnóstica de la gestante en los servicios de salud, y actuación del enfermero en ese proceso.

\section{MATERIAL Y MÉTODOS}

Para alcanzar el objetivo propuesto, se optó por realizar una investigación bibliográfica, pues se refiere a una recogida general de los principales trabajos ya realizados, proporcionando datos sobre determinado asunto, poniendo a los investigados en contacto directo con todo lo que fue escrito sobre el tema en estudio ${ }^{8}$. 
Se realizó una investigación en bases de datos especificamente ofrecidas por Scielo utilizándose como descriptores las siguientes palabras: Enfermería, VIH, AIDS, Mujeres.

\section{RESULTADOS DE DISCUSIÓN}

Se encontraron 56 publicaciones, de las cuales tras realizar una lectura exploratoria de sus resúmenes, se seleccionaron 17 artículos que contienen el tema propuesto.

Tabla 1: Fuentes virtuales investigadas relacionadas con la gestante y el HIV/AIDS

\begin{tabular}{lcc}
\hline Fuentes & Número de Artículos & Frecuencia (\%) \\
\hline Lilacs & 17 & 100 \\
Medline & 0 & 0 \\
Scielo & 0 & 0 \\
Total & 17 & 100 \\
\hline
\end{tabular}

Tabla 2. Año de las publicaciónes relacionada con la gestante y el HIV/AIDS.

\begin{tabular}{ccc}
\hline Año de Publicación & Número de Artículos & Frecuencia $(\%)$ \\
\hline 2008 & 1 & 5,9 \\
2007 & 4 & 23,5 \\
2006 & 4 & 23,5 \\
2005 & 1 & 5,9 \\
2004 & 0 & 0 \\
2003 & 2 & 11,8 \\
2002 & 2 & 11,8 \\
2001 & 1 & 5,9 \\
2000 & 1 & 5,9 \\
1999 & 0 & 0 \\
1998 & 1 & 5,9 \\
Total & 17 & 100 \\
\hline
\end{tabular}

Los años 2006 y 2007 fueron los que tuvieron el mayor número de publicaciones, monstrándonos que se trata de um tema bastante actual, conforme se verifica en la tabla 2.

Tabla 3. Fuente impresa.

\begin{tabular}{lcc}
\hline \multicolumn{1}{c}{ Fuente impresa } & Número de Artículos & Frecuencia (\%) \\
\hline $\begin{array}{l}\text { Revista Latino Americana } \\
\text { de Enfermagem }\end{array}$ & 4 & 23,5 \\
$\begin{array}{l}\text { Revista Brasileira de } \\
\text { Enfermagem }\end{array}$ & 3 & 16,6 \\
$\begin{array}{l}\text { Revista Brasileira de } \\
\begin{array}{l}\text { Saúde Materno Infantil } \\
\text { Acta Paulista de }\end{array}\end{array}$ & 4 & 23,5 \\
$\begin{array}{l}\text { Enfermagem } \\
\text { Revista Escola de } \\
\text { Enfermagem da USP Nery }\end{array}$ & 1 & 5,9 \\
$\begin{array}{l}\text { Revista Escola Anna Nery } \\
\text { Texto Contexto - }\end{array}$ & 1 & 11,8 \\
Enfermagem & 1 & 5,9 \\
\end{tabular}


Revista Brasileira de

Ginecologia e Obstetrícia

Total

17

100

Las revistas que más artículos disponibles tuvieron sobre el tema propuesto fueron la Revista Brasileira de Saúde Materno Infantil y la Revista Latino Americana de Enfermagem, conforme observado en la tabla arriba.

Tabla 4. Localidad de las publicaciones investigadas.

\begin{tabular}{lcc}
\hline Lugar & Número de artículos & Frecuencia (\%) \\
\hline Brasília & 3 & 16,6 \\
São Paulo & 3 & 16,6 \\
Ribeirão Preto & 4 & 23,5 \\
Rio de Janeiro & 1 & 5,9 \\
Recife & 4 & 23,5 \\
Botucatu & 1 & 5,9 \\
Florianópolis & 1 & 5,9 \\
Total & 17 & 100 \\
\hline
\end{tabular}

La ciudad que tuvo mayor número de publicaciones fue Ribeirão Preto, mostrándonos que hay una mayor preocupación en aquella región sobre el tema.

Tabla 5. Idioma de las publicaciones consultadas.

\begin{tabular}{lcc}
\hline Idiomas & Número de Artículos & Frecuencia (\%) \\
\hline Portugués & 17 & 100 \\
Total & 17 & 100 \\
\hline
\end{tabular}

Solamente se investigaron artículos en portugués, ya que mostramos las estrategias utilizadas en Brasil.

Tabla 6: Métodos de publicaciones

\begin{tabular}{lcc}
\hline MÉTODOS & FRECUENCIA & FRECUENCIA (\%) \\
& ABSOLUTA & \\
\hline CUALITATIVO & 15 & 88,2 \\
CUANTITATIVO & 2 & 11,8 \\
TOTAL & 17 & 100 \\
\hline
\end{tabular}

El método más utilizado fue el cualitativo, pues se trata de un tema bastante complejo, siendo desta forma inviable cuantificarlo.

Tabla 7. Resultados comunes de los estudios pesquisados.

\begin{tabular}{|c|c|c|}
\hline Resultados & Número de Artículos & Frecuencia (\%) \\
\hline $\begin{array}{l}\text { Adhesión a la terapia } \\
\text { antirretroviral }\end{array}$ & 3 & 16,6 \\
\hline Gestante y el HIV & 5 & 29,4 \\
\hline Transmisión Vertical & 3 & 16,6 \\
\hline $\begin{array}{l}\text { Pacientes portadores de } \\
\text { HIV/AIDS }\end{array}$ & 3 & 16,6 \\
\hline $\begin{array}{l}\text { Centro de diagnóstico y } \\
\text { cuidado }\end{array}$ & 3 & 16,6 \\
\hline Total & 17 & 100 \\
\hline
\end{tabular}


Trs el análisis de los artículos se llevaron a cabo las siguientes estrategias para la detección y prevención de la transmisión vertical del virus VIH.

\section{Evaluación y educación sexual del VIH}

Se percibe a través de la lectura de los artículos que la detección precoz del VIH debe ocurrir principalmente en el nivel primario de atención, o sea, en las unidades básicas de salud de la familia, ya que hay una facilidad en la identificación de esas pacientes, pues hay una cobertura más eficaz de las familias atendidas. El enfermero, como integrante del equipo de salud de la familia, debe estar atento, ofreciendo medios para la identificación de esas gestantes, a través de las visitas domiciliarias de los agentes comunitarios de salud, una vez que estos profesionales están capacitados y entrenados por el enfermero de la unidad de salud. Este proceso de busca de las gestantes debe formar parte del cotidiano del profesional, a fin de evitar la transmisión vertical.

El Ministerio de Salud normatizó como rutina de atención prenatal el ofrecimiento del examen anti-VIH, ya que la solicitud y realización del test anti-VIH en la gestación favorece la identificación de portadoras del virus y la posibilidad de consejo precoz para tratamiento y acompañamiento adecuados de la gestante. Siendo realizado con el consentimiento verbal de la gestante, teniendo como requisito básico sesiones de apoyo pre y post test. Este se pauta especialmente en un proceso de escucha de las demandas, intercambio de informaciones y apoyo emocional. Se configura en un diálogo tendente a establecer una relación de confianza entre los interlocutores y ofrecer a la gestante condiciones para que evalúe su condición de vulnerabilidad y riesgos personales de portar el $\mathrm{VIH}$, tome decisiones y encuentre maneras realistas, 0 sea, maneras viables de enfrentar sus problemas relacionados con el HIV/AIDS. Un estudio apunta que, para pacientes con DST, el consejo mejora los índices de adhesión al tratamiento, el retorno para recibir el resultado de los exámenes, el tratamiento del compañero sexual y uso del preservativo durante el tratamiento ${ }^{1,9,10}$.

Uno de los aspectos que pueden contribuir a una buena cobertura de evaluación del VIH en el prenatal es la realización de una educación para la salud de calidad. Esa calidad se traduce especialmente en la escucha de las demandas de las usuarias, en el apoyo emocional para ayudarlas a enfrentar los conflictos que normalmente aparecen a causa de la evaluación y en el intercambio de informaciones. Así, la educación para la salud, como una estrategia de prevención, formula discursos que mueven a la reflexión, superación de dificultades en el enfrentamiento de los problemas relacionados con HIV/AIDS y adopción de medidas preventivas en la busca de una mejor calidad de vida. El acceso a las acciones de la educación para la salud es un derecho de toda gestante/parturienta, posibilitando el conocimiento y, consecuentemente, la correcta adhesión al tratamiento 1, 9, 10,11.

\section{Adhesión a la terapia antirretroviral tras el diagnóstico de seropositividad}

Desde el inicio de la epidemia de AIDS o SIDA en los años ochenta hasta hoy, mucho se ha hecho para proporcionar mejor calidad de vida a los portadores del $\mathrm{VIH}$, entre otras acciones, está la distribución universal de medicamentos, resaltándose que Brasil fue el primer país del tercer mundo en adoptar tal medida Estudios muestran 
que la tasa de transmisión vertical del VIH, sin intervención, se sitúa en torno al $20 \%$, $\mathrm{y}$, con las medidas tomadas, se redujo a cero y $2 \%{ }^{9,11,12}$.

La no adhesión a los nuevos medicamentos para el AIDS (anti-retrovirales - ARV) es considerada como uno de los más amenazadores peligros para la efectividad del tratamiento, en el plano individual, y para la propagación de virus-resistencia, en el plano colectivo. Esto porque los nuevos regimes terapéuticos parecen exigir del individuo que se adhiere al tratamiento integración compleja entre conocimientos, habilidades y aceptación, además de otros importantes factores ligados al ambiente y al cuidado a la salud ${ }^{11,12,13}$.

Los datos encontrados en la literatura sobre los factores de riesgo para la no adhesión a los tratamientos de enfermedades crónicas en general, así como de infección por $\mathrm{VIH}$, no son concluyentes. Algunos factores son considerados relevantes, sin embargo es fundamental que los profesionales que actúan con personas infectadas por VIH conozcan cuáles son los factores de riesgo de no adhesión a la terapia ${ }^{12,13,}$ 16,17

Estudios demuestran que es fundamental que haya una interacción entre el cliente/cuidador y el equipo multiprofesional, para favorecer que la complejidad que envuelve la continuidad del tratamiento pueda ser debidamente aclarada, permitiendo de esta forma el esclarecimento de las dudas y la continuidad del tratamiento. Algunos estudios demuestran también que la actuación del enfermero debe ser de aclarar a los pacientes sobre su estado de salud, revelando de esta forma un tratamiento más holístico, en la medida que este pasa a "involucrarse" más con el paciente, la enfermería debe tener un interés especial en las necesidades individuales para el autocuidado y su provisión y organización en una base continua, de modo a sustentar la vida y la salud, recuperarla de la enfermedad e incentivarla a luchar contra sus efectos $6,11,13,16$.

La enfermería es una profesión que no exige solamente el conocimiento de un conjunto de técnicas específicas, pues en todos los sectores, son solicitados a oferecer un cuidado integral, abarcando los aspectos biológicos, psicosociales y espirituales, principalmente, quando este es para pacientes con enfermedades crónicas. Siendo imprescindible el acompañamiento de este profesional a los pacientes portadores de HIV/AIDS, atendiendo a su bienestar y a incentivar la adhesión de terapia medicamentosa, en especial, a las gestantes, pues se sabe que la detección precoz, así como el uso de los medicamentos disminuyen significativamente la posibilidad de la transmisión vertical ${ }^{2,6,11,14}$.

En un estudio realizado con gestantes, para identificar los factores de la no adhesión al tratamiento, se encontró que no solo contribuía un factor sino varios, interligados en su día a día, en lo que se refiere a las dificultades y enfrentamiento de su cotidiano ${ }^{9}$

El equipo de enfermería debe estar articulado para ofrecer una intervención transformadora, actuando en el foco de las varias especificidades que llevan a las gestantes a no adherirse a la terapia. La comprensión del trabajo desarrollado por este equipo puede contribuir a una actuación específica en los puntos identificados como sensibles y perjudiciales para el buen desarrollo del proceso de la terapia medicamentosa. 


\section{CONSIDERACIONES FINALES}

El estudio nos permitió un panorama de las estrategias utilizadas en la detección del HIV/AIDS en las gestantes, así como el tratamiento de las mismas, a fin de evitar la transmisión vertical del virus.

Se encontró como estrategia utilizada en las unidades básicas de salud, en los centros de evaluación y educación sexual (CTA) y en los Hospitales en general, la realización del test anti-VIH, acompañado de un protocolo que incluye la educación pre y post test. Se percibió que el enfermero tiene significativa importancia en ese proceso, siendo la mayoría de las veces el principal responsable del acompañamiento. Demostrándonos nuestra responsabilidad con la vida, además de cumplir con los principios de la enfermería aprendidos durante el curso: Educar, Cuidar e Investigar.

Otra estrategia encontrada fue la adhesión a la terapia medicamentosa anti-retroviral, donde percibimos que hay una incidencia muy grande de pacientes que no siguen correctamente las orientaciones o que incluso abandonan esa terapia. Los motivos apuntados son innumerables, sin embargo, se determinó que el profesional responsable, en especial el enfermero, debe estar atento a las dificultades de cada paciente, como único, permitiendo de esta forma crear intervenciones especificas para cada dificultad, volviendo a la atención humanizada, basada en la ética y en el compromiso con la vida humana.

Por tanto, los servicios de salud deben estar organizados para realizar esa detección y tratamientos precozmente y la enfermera, así como los demás profesionales del equipo, deben estar involucrados en la busca de solucciones para las transformaciones que la infección por el VIH trajo para la asistencia a la salud de la gestante, del feto y de la familia. Son varios los desafíos encontrados, siendo necesaria la constante actualización para la asistencia de enfermería, constituyendo la aplicación de acciones y de informaciones científicas con el objetivo de la prevención y tratamiento abarcando el aprendizaje individual y colectivo, motivador de la comprensión y de la consciencia para el establecimiento de estrategias fundamentadas en el conocimiento.

\section{REFERENCIAS}

${ }^{1}$ Maria ALA, Francisca LRF, Alanna VBR. Aconselhamento pós-teste anti-HIV: análise à luz de uma teoria humanistica de enfermagem. Esc. Anna Nery v.10 n.3 Rio de Janeiro dez. 2006

2 Lis ASN, Elucir G. Mães portadoras de HIV/AIDS: percepções acerca da severidade da infecção. Rev. esc. enferm. USP v.41 n.4 São Paulo dez. 2007

3 Edilene LM, Neide SP. Transmissão vertical do HIV: expectativas e ações da gestante soropositiva.Revista Latino-Americana de Enfermagem v.14 n.3 Ribeirão Preto maio/jun. 2006

${ }^{4}$ Geraldo D, Carla VC, Alessandra CM, Marina CP, Silvana MQ, Marissa MMP. Teste rápido para detecção da infecção pelo HIV em gestantes. Rev. Bras. Ginecol. Obstet. v.23 n.2 Rio de Janeiro mar. 2001

${ }^{5}$ Léa MMB, Marli TGG. Avaliação de atendimento prestado por profissionais de saúde a puérperas com HIV/AIDS. Texto contexto enferm. v.16 n.3 Florianópolis jul./set. 2007 
${ }^{6}$ Juliana PC, Lucilane MSS, Maria RFS,Karla CLM. Expectativas de pacientes com HIV/AIDS hospitalizados, quanto à assistência de enfermagem. Revista Brasileira de enfermagem v.59 n.2 Brasília mar./abr. 2006

${ }^{7}$ Kátia VOF, Maria HK. As necessidades comunicacionais das práticas educativas da prevenção da transmissão materno-fetal do HIV. Rev. Bras. Saude Mater. Infant. v.3 n.4 Recife out./dez. 2003

${ }^{8}$ Marconi MA, Lakato EM. Técnicas de pesquisa. Editora Atlas, São Paulo, 2002

${ }^{9}$ Petrolina LC, Fernanda RIP, Lisiane MQ. Gestantes HIV positivas e sua não-adesão à profilaxia no pré-natal. Rev. bras. enferm. v.60 n.5 Brasília set./out. 2007

10 Vânia S, Dina C. Considerações sobre os discursus de aconselhamento nos centros de testagem anti- HIV. Interface (Botucatu) v.11 n.23 Botucatu set./dez.2007

${ }^{11}$ Maria JRV, Sônia MOB. Redução da transmissão vertical do HIV: Desafio para a assistência de enfermagem Rev. Latino-Am. Enfermagem v.8 n.2 Ribeirão Preto abr. 2000

${ }^{12}$ Maria Rosa Ceccato Colombrini'; Maria Helena Baena de Moraes Lopes"; Rosely Moralez de Figueiredo"lI Adesão à terapia antiretroviral para HIV/AIDS Rev. esc. enferm. USP v.40 n.4 São Paulo dez. 2006

${ }^{13}$ Elucir G, Carla GV, Marcela DO Adesão à terapêutica anti-retroviral por indivíduos com HIV/AIDS assistidos em uma instituição no interior paulista. Rev. Latino-Am. Enfermagem v.13 n.5 Ribeirão Preto set/out, 2005

${ }^{14}$ Petronila LC, Lucilda S. Mulheres com HIV/AIDS: fragmentos de sua face oculta. Revista Brasileira de enfermagem v.60 n.2 Brasília mar./abr. 2007

${ }^{15}$ Neide SP, Maria RDOL Saúde sexual e reprodutiva com enfoque na transmissão do HIV: práticas de puérperas atendidas em maternidades filantrópicas do município de São Paulo. Revista Brasileira Saúde Materno Infantil v.3 n.1 Recife jan./mar. 2003

16 Maria JRV, Sônia MOB. Gestantes infectadas pelo HIV - Caracterixação e diagnóstico de enfermagem. Acta Paulista de Enfermagem v.15, abr/jun, 2002

17 Marcos VOL, Maria NOF. Pessoas vivendo com HIV: estrsse e suas formas de enferntamento. Rev. Latino-Am. Enfermagem v.6 n.4 Ribeirão Preto out. 1998

ISSN 1695-6141

(C) COPYRIGHT Servicio de Publicaciones - Universidad de Murcia 